\title{
Agentes Conversacionais: Desenvolvimento de uma Aplicação no Museu Virtual para o Ensino de Computação
}

\author{
${ }^{1}$ Andreia S.Bos ${ }^{1}$, Giliane Bernardi ${ }^{3}$, Felipe Muller ${ }^{3}$, Lucas Plautz Prestes ${ }^{1}$ Milton \\ A. Zaro ${ }^{1}$ Michelle Pizzato ${ }^{2}$ \\ ${ }^{1}$ Centro Interdisciplinar de Novas Tecnologias na Educação- Universidade Federal do \\ Rio Grande do Sul (UFRGS) \\ CEP - 90040-060- Porto Alegre - RS - Brazil \\ ${ }^{2}$ Instituto Federal do Rio Grande do Sul (IFRS) Porto Alegre - RS - Brazil \\ CEP - 90030-041- Porto Alegre - RS - Brazil \\ ${ }^{3}$ Departamento de Computação Aplicada - Universidade Federal de Santa Maria \\ (UFSM) Av. Roraima 1000 - Santa Maria - RS - Brasil \\ \{andreia.bos,bgiliane; lucas.plautz.prestes\}@gmail.com, zaro@ufrgs.br, \\ michelle.pizzato@poa.ifrs.edu.br; felipedinf.ufsm.br
}

\begin{abstract}
Virtual 3D worlds are spaces where students of Computer Licensing can have the support of software agents to simulate avatars in the accomplishment of tasks. The contribution of this article is the aggregation of knowledge and bot conversation, which communicates with students through a natural language. To this end, we use the AIML (Artificial Intelligence Markup Language) language to allow us to perform some precise conversations about teaching introduction to computing. In this proposal we evaluate the usability of the conversational agent in a 3D Virtual Museum. The results show the conversation approach and show greater student satisfaction using bot intelligence.
\end{abstract}

Resumo. Mundos Virtuais $3 D$ são espaços em que os estudantes de Licenciatura em Computação podem ter o apoio de agentes de software para simulação de avatares na realização de tarefas. A contribuição deste artigo é a agregação de conhecimento e a conversação do bot, que se comunique com os estudantes por meio de uma linguagem natural. Com este objetivo, usamos da linguagem AIML (Artificial Intelligence Markup Language) para nos permitir realizar algumas conversas precisas sobre o ensino de introdução à computação. Nessa proposta avaliamos a usabilidade do agente conversacional em um Museu Virtual 3D. Os resultados mostram a abordagem da conversação e apresenta uma maior satisfação do estudante utilizando a inteligência do bot.

\footnotetext{
${ }^{1}$ Andréia Solange Bos, Doutoranda UFRGS;Prof ${ }^{\mathrm{o}}$ Dr. Felipe Muller-UFSM; Prof $^{\mathrm{a}}$ Dr $^{\mathrm{a}}$ Giliane BernardiUFSM; Lucas Plautz Prestes, Doutorando UFRGS, Prof ${ }^{\circ}$ Dr. Milton Zaro- UFRGS; Prof ${ }^{\mathrm{a}}$ Dr $^{\mathrm{a}}$. Michelle Pizzato IFRS.
} 
VII Congresso Brasileiro de Informática na Educação (CBIE 2018)

Anais dos Workshops do VII Congresso Brasileiro de Informática na Educação (WCBIE 2018)

\section{Introdução}

O presente artigo visa corroborar com Graesser e Charlie sobre a utilização de agentes inteligentes como tutores em um ambiente imersivo. Os mundos imersivos, se caracterizam por serem ambientes criados e definidos com objetivo de reunir pessoas de interesses em comum a fim de trocar conhecimento e experiências de forma colaborativa por meio de mecanismos de comunicação síncronos e assíncronos, tais mundos tem como diferencial possibilitar a manipulação de objetos em 3D criarem novas comunidades ou subdivisão em grupos menores conhecidos como subcomunidades.

Os Mundos Virtuais em 3D são ambientes virtuais criados e definidos como espaços em que as pessoas interagem umas com as outras a fins de socializar e encontrar um entretenimento. Com o avanço da internet e a cada dia sendo mais interativa envolvem as pessoas em experiências digitais em 3D para fins recreativos e outros objetivos. Podemos citar o uso na educação, colaboração, e por permitir a interação entre vários usuários on line e podermos personificar usando agentes de software. Com base nestas abordagens um sistema de computador, ou seja um agente de software pode ter uma combinação com as interfaces em 3D, estes sistemas podem permitir que os humanos executam tarefas complexas específicas proporcionando uma imersão nos mundos virtuais e a interação com os outros participantes.

As instituições definem um modelo para definirem uma estrutura, para que haja interação dos participantes, definindo regras para que ocorra a comunicação. Em nosso caso nos baseamos na linguagem AIML, para agregar conhecimento específico ao bot. Quando o Mundo Virtual é executado, podemos visualizar os estudantes que estão logados imersos no ambiente. Podemos observar usuários humanos sendo avatares e realizando o percurso virtual. Quanto aos Mundos Virtuais, estes podem proporcionar cenários envolventes, no qual os usuários participam das interações uns com os outros. Um agente NPC personificado pode ser usado como um bot (uma representação do humano) para atingir os objetivos como um agente. Assim a interação do usuárioagente é fundamental para que ele se sinta como sendo vigiado por um agente companheiro. No entanto, a interação baseada em script é propenso a erros, especialmente para usuários que não estão familiarizados com sistemas baseados em comandos. Interações de linguagem Natural constituem em uma alternativa adequada para sistemas baseados em comandos. Os agentes de conversação são protótipos para este tipo de interação. Estes são personagens virtuais que são capazes de se envolver em uma conversa com os seres humanos.

Hoje em dia podem ser encontrados principalmente como assistentes virtuais prestando informações aos usuários em ambiente web, especificamente a partir de conversas dos bots em AIML. Os bots reagem e são conhecidos pelas estruturas de diálogo definidas em arquivos estáticos. Estes são projetados para fornecer, quando solicitado, informações aos usuários em geral. Eles são capazes de pedir informações gerais do usuário ( por exemplo, o seu nome, ou em que posso te ajudar) e busca essa informação em sua base de conhecimento. A contribuição deste trabalho é o uso de técnicas de interações de conversação através de agentes de software em mundos virtuais. Em particular os consagrados bots interagindo com os seres humanos através de mecanismos de interação . O bot só entende as mensagens do usuário na língua 
VII Congresso Brasileiro de Informática na Educação (CBIE 2018)

Anais dos Workshops do VII Congresso Brasileiro de Informática na Educação (WCBIE 2018)

portuguesa. O mecanismo de interação é avaliada em termos da eficácia e eficiência do usuário, seus erros e satisfação quanto a conversação.

\section{Mundos Virtuais 3D}

Ambientes 3D imersivos caracterizam os chamados Mundos Virtuais 3D, ou simplesmente mundos virtuais. Cada usuário participa dos mundos virtuais através de um avatar que pode ser personalizado por uma representação gráfica de si próprio imerso no ambiente, por meio do qual pode se movimentar naquele espaço, como se estivesse fisicamente caminhando, ou ocorrendo o ato de voar no ambiente (Morgado, 2011).

Mundos Virtuais são ambientes eletrônicos que emulam espaços físicos complexos através de representações de ambientes simulados, contendo objetos e interações com seus avatares (Mennecke, 2008)

Em um Mundo Virtual 3D, o usuário é caracterizado como um avatar. Na área da computação, avatar é considerado um cibercorpo inteiramente digital, uma figura gráfica de complexidade variada que empresta sua vida simulada para o transporte de cibernautas para dentro dos mundos paralelos do ciberespaço. O usuário é situado, vê e ouve coisas daquele ponto de vista e então com a simulação parece estar presente naquele ambiente (Guomin, Jianxin, 2010).

Para chegar ao conceito de Mundo Virtual, Morgado (2011) utiliza o conceito de ambiente virtual, ou seja "tudo aquilo em que agimos sem se poder tocar diretamente com as mãos, o que a tecnologia eletrônica nos mostra e só existe fisicamente sob a forma de posições, velocidades em conjunto com conceitos de multiutilização e de presença imersiva. Diante destas assertivas, apresenta o conceito de Mundo Virtual como:

Plataformas computacionais (jogos ou ambientes sociais), onde podem estar telepresentes imersivamente vários jogadores ou utilizadores, que podem comunicar-se entre si. Ou seja, a participação desses utilizadores no mundo dá-se através de uma personagem virtual ("avatar") e - aspecto fundamental - é através deste avatar que o utilizador interage com o mundo e por ele é afetado (Morgado, 2011, p. 6).

Para Guomin e Jianxin (2010), ambientes imersivos são os espaços que utilizam sistemas computacionais para promover um estado de ilusão perceptiva e que incitam o visitante à participação. A imersão é usada como um estágio para aniquilar a diferença entre realidade e representação e como instrumento de persuasão da mente fazendo a passagem entre o realismo e o fantasioso. Neste caso, é possível considerar como representações a partir dos mundos que imaginamos.

"um mundo virtual, no sentido amplo, é um universo de possíveis, calculáveis a partir de um modelo digital. Ao interagir com o mundo virtual, os usuários o exploram e o atualizam simultaneamente. Quando as interações podem enriquecer ou modificar o modelo, o mundo virtual torna-se um vetor de inteligência e criação coletiva" (Guomin e Jianxin (2010). 
VII Congresso Brasileiro de Informática na Educação (CBIE 2018)

Anais dos Workshops do VII Congresso Brasileiro de Informática na Educação (WCBIE 2018)

A seguir são apresentadas as plataformas para criação de mundos virtuais.

\section{Plataforma de criação de Mundos Virtuais}

Os mundos virtuais 3D construídos podem ser divididos em dois tipos: os de plataforma proprietária e os de plataforma aberta. Os mundos de plataforma proprietária são de uma empresa controladora que determina as condições de uso do mesmo, autorizando ou não os usuários a realizarem modificações. Já os de plataforma aberta têm seus códigos fontes disponibilizados para que usuários possam realizar modificações ou correções no software (Carmo, 2013).

Dentre os mundos virtuais 3D existentes, destacam se plataformas proprietárias o Second Life e o Active Worlds, plataformas abertas o OpenSimulator e o Open Wonderland. O desenho, implementação e manutenção desses sistemas open_source, geralmente, são feitos por grupos de entusiastas desenvolvedores, que podem ser apoiados por fundações.

Para o desenvolvimento deste trabalho foi escolhido o mundo virtual 3D OpenSimulator, um ambiente 3D de código aberto, compatível com o Second Life. Tais características foram consideradas para a sua escolha, pois, além de ser de plataforma aberta, sua compatibilidade com o Second Life facilita a busca por modelos 3D e exemplos de scripts importantes no desenvolvimento do projeto. Além disso, esta é a plataforma que já vem sendo utilizada pelo grupo de pesquisa no qual os autores estão inseridos. A seguir é apresentado os agentes de software.

\section{Agentes de Software}

Esta seção tem por objetivo abordar sobre o desenvolvimento e a utilização de agentes inteligentes em Mundos Virtuais 3D. Inicialmente serão destacados alguns conceitos e características sobre os mesmos.

Para Russel e Norvig (2004), um agente é algo que percebe e age em um ambiente, sendo capaz de perceber o seu ambiente por meio de sensores e de agir sobre esse ambiente por intermédio de atuadores.

Wooldridge (2009) conceitua agente como um programa que auxilia o usuário na realização de alguma tarefa ou atividade. Agentes inteligentes tratam-se basicamente de um sistema capaz de tomar decisões e interagir com o ambiente ou outros usuários com base em alguma fonte de dados. Todo agente inteligente deve apresentar ao menos quatro características (Wooldridge, 2009):

1. Autonomia, possuir controle sobre suas ações;

2. Reatividade, perceber alterações ao seu redor;

3. Sociabilidade, interagir com outros agentes através de algum tipo de linguagem de comunicação;

4. Pró - atividade, não só reagindo ao ambiente, mas tomando iniciativas quando conveniente. 
VII Congresso Brasileiro de Informática na Educação (CBIE 2018)

Anais dos Workshops do VII Congresso Brasileiro de Informática na Educação (WCBIE 2018)

O termo agente inteligente pode ser usado para referir-se a um agente de software que possui alguma inteligência. A inteligência artificial tem exercido uma influência muito forte sobre o campo dos agentes e já evoluiu muito (Wooldridge, 2009). A seguir é descrito os chatterbots.

\subsection{Chatterbots}

Os chatterbots são agentes inteligentes de computadores que utilizam algumas técnicas de inteligência artificial para simular conversas com pessoas reais. A simulação é realizada através de trocas de mensagens de textos ou áudios, como se o usuário estivesse em um bate-papo virtual (Teixeira, 2005).

Esse tipo de agente processa uma entrada em linguagem natural e consulta uma base de conhecimento para dar respostas que imitam as respostas humanas. Uma vantagem dos chatterbots é o grande poder de interação. Na educação podem ser potenciais para o uso como um método de ensino mais inovador e eficiente, em interagir com a máquina (Mahapatra, 2012). Na sequência é mostrada a linguagem AIML utilizada no Chatterbot.

\subsection{Linguagem AIML}

A Linguagem AIML (Artificial Intelligence Markup Language) é uma linguagem de marcação, baseada em XML (eXtensable Markup Language) e utilizada na base de conhecimento de robôs de conversação, e é baseada na técnica de casamento de padrões. O projeto A.L.I.C.E (Wallace, 2001) é baseado nessa técnica.

A linguagem AIML descreve uma classe de objetos, conhecidos como objetos AIML, que são interpretados por um programa de computador que descreve o comportamento do robô de conversação. Para o funcionamento do chatterbot é necessário um interpretador da linguagem AIML, sendo que este interpretador, disponível em diversas linguagens, reconhece as principais tags de AIML.

\section{Trabalhos Relacionados}

$\mathrm{Na}$ literatura podemos encontrar muitos sistemas de conversação. Destes destacam-se os sistemas tutores, que são os tutores virtuais que seguem princípios pedagógicos (Mikic, 2009). O tutor virtual é um personagem 3D com interface textual que armazena o seu conhecimento em um currículo e usa um algoritmo de reconhecimento de padrões. Os autores realizam avaliações empiricamente de alunos aprendendo e a qualidade do diálogo. Além disso, nosso objetivo é oferecer suporte aos usuários simulando um humano.

Charlie, 2009 usa de mecanismos AIML(Artificial Intelligence Markup) para manter uma conversa geral com os alunos. A interface de usuário com o bot é uma janela pop-up com área de texto dedicado a conversa. Especificamente determinadas palavras-chave relacionadas com modelos AIML são utilizadas pelos estudantes e após isso geram conversas personalizadas. Do mesmo jeito, nosso bot é baseado em AIML, 
VII Congresso Brasileiro de Informática na Educação (CBIE 2018)

Anais dos Workshops do VII Congresso Brasileiro de Informática na Educação (WCBIE 2018)

mas eles expandiram a linguagem que visa realizar tarefas. Além disso nossa interface é um Mundo Virtual simulando ambientes virtuais 3D e fornece uma experiência imersiva para usuários humanos.

Ainda Graesser, 2005 desenvolve a personalidade de chat-bots para alterar o tratamento para utilizadores de acordo com a sua atitude e humor. Galvão, 2004 desenvolveu o Persona-Aiml, que foi testado em um aplicativo independente e na rede.

Ainda o trabalho de Mori, 2003 propõe um módulo de conversa baseado na web que detecta temas solicitado pelo usuário e faz uma transição entre tópicos por avisar o usuário. Nossa extensão da AIML inclui também marcas para apoiar conversas para o usuário. Nosso bot verifica as respostas do usuário com base na especificação e em sua base de conhecimento específica. Além disso, estas obras têm interfaces baseadas na web, ao contrário de nossa interface que é desenvolvida em um mundo virtual 3D, gerenciando conversas simultâneas com outros usuários.

$\mathrm{Na}$ linha de ambientes virtuais 3D Kluwer 2012, implementa um barman como um personagem $3 \mathrm{D}$ conversacional em um jogo 3D on line multiplayer de compras em massa. O barman recomenda e vende bebidas para os clientes, e também realiza um diálogo social com pequenas conversas, tem o seu conhecimento baseado em ontologias adquiridas através da aplicação de tecnologia web semântica. O componente de compreensão da linguagem Natural usa uma memória de contexto e um estado de diálogo, e a entrada do usuário é processada para após selecionar a resposta adequada. A principal diferença com a nossa abordagem é que enquanto Komparse se destina a entreter os usuários em um determinado jogo, o nosso objetivo é fazer com que o bot seja um agente companheiro em suas atividades de ensino.

\section{Desenvolvimento do Agente}

Para o desenvolvimento foi implementado uma interface em 3D, representando um espaço imersivo, em que os usuários podem interagir de forma intuitiva e seguir em um percurso para realização de atividades que eles estão envolvidos. Os utilizadores humanos são representados por avatares conforme mostra a figura 1:

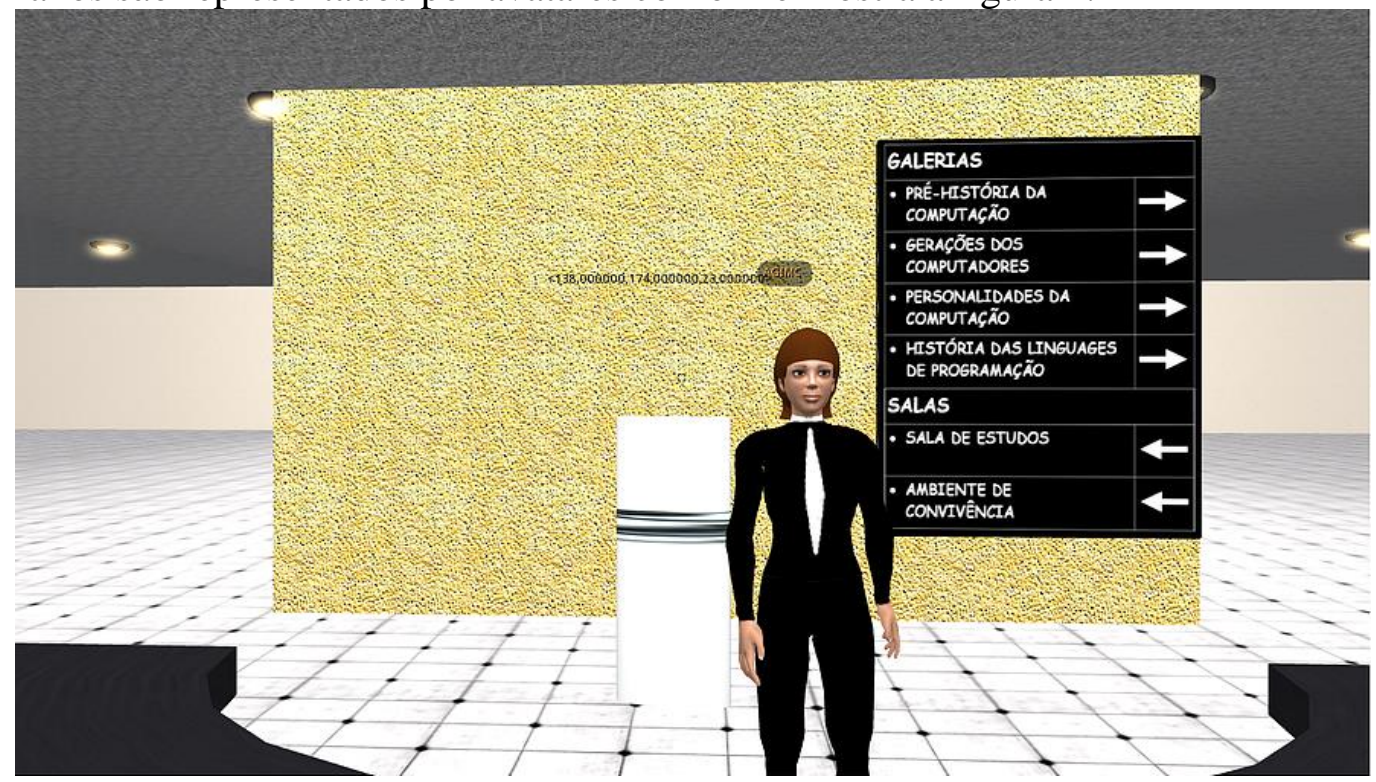

Figura 1. Agente no Museu da Computação 
VII Congresso Brasileiro de Informática na Educação (CBIE 2018)

Anais dos Workshops do VII Congresso Brasileiro de Informática na Educação (WCBIE 2018)

O sistema é baseado na linguagem AIML e utiliza um bot de conversação. Quando o usuário realiza uma pergunta ao agente, o mesmo busca e interpreta em sua base de conhecimento para transformar em linguagem natural e retorna a resposta ao usuário. Nosso foco está no sistema de conversação, nos diálogos e na linguagem natural, em que os usuários podem expressar suas intenções de maneiras diferentes e os bots tem que entender o que dizem os usuários e assim apoiá- los na lição, sendo um complemento para a sala de aula. Para este fim o bot deve perguntar ao usuário as suas dúvidas.

$\mathrm{O}$ agente responde em linguagem simples e natural, quando é solicitado pelo usuário. Na verdade AIML é um dialeto XML que tem conhecimento de conversação encapsulada em objetos e dados chamados de categorias. Basicamente cada categoria é definida por uma entrada do usuário ou questão de entrada. As categorias são agrupadas em tópicos e podem especificar uma mudança de assunto. Portanto designer de bots pode encapsular conhecimento de conversação por tópicos e definir fluxos de conversas. Apesar de sua simples estrutura e funcionamento, os robôs de conversação AIML pode dar respostas para quase qualquer frase que o usuário imaginar, é uma questão de estender o conhecimento da conversa com mais e mais arquivos AIML.

No entanto AIML também pode funcionar em ambientes 3D no qual é preciso estruturar internamente um bot, e ter habilidades básicas de conversação para acolher o usuário. Ele precisa ser proativo a fim de gerenciar conversas e dar suporte ao usuário no desempenho de sua visita virtual. A seguir é mostrada os detalhes da estrutura e implantação das conversas. A primeira fase da conversa é as boas vindas e em que posso te ajudar para o usuário começar a interagir. Na Figura 2 é ilustrada a conversação de boas vindas.

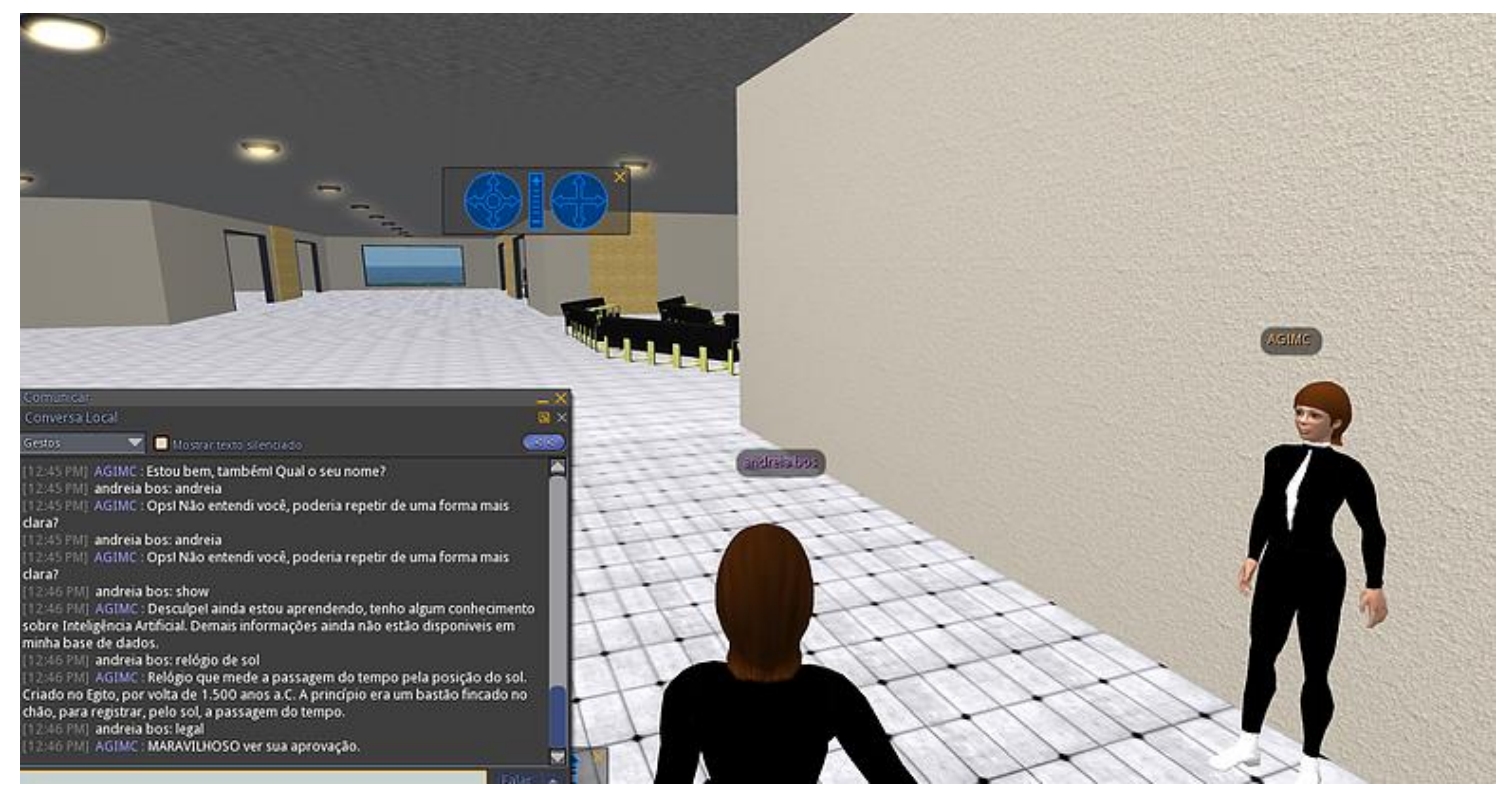

Figura 2. Interação do Agente

A estrutura da conversa é realizada em duas etapas: as de boas-vindas e interações sociais e a outra etapa com conhecimentos específicos sobre o conteúdo da base de conhecimento. A estrutura da conversa de boas vindas e despedidas é articulada da mesma base de conhecimento junto com as interações sociais. O tema é acolhedor dando ao usuário uma ilusão de estar com um agente companheiro. Os tópicos que 
foram inseridos na base de conhecimento são padrões AIML e são usados para cumprimentar e se despedir dos usuários, informando-os sobre determinadas galerias que eles podem fazer o acesso. Este fluxo segue uma estrutura definida pelo conhecimento do bot.

O primeiro tipo de interação ocorre após o reconhecimento de que existe um usuário para ele interagir. Este reconhecimento é feito através de um comando que o usuário executa, sendo /create e o mesmo pode começar a interagir. Isto é feito utilizando categorias padrões AIML que redirecionam entradas específicas, contendo certas palavras-chave dos usuários como um tópico a ser respondido. Neste ponto o bot toma a iniciativa e pede ao usuário em que posso te ajudar? , e retorna a sugestão para o usuário. Se a entrada do usuário é inválida, ou seja, um caracter que o bot não reconhece, ele retorna ao usuário e informa "Você poderia repetir a sua pergunta de uma forma mais clara", fazendo com que o usuário retorne a pergunta, para continuar a sua interação. O bot é, portanto um autômato e seleciona a próxima ação para executar com base nas entradas do usuário.

Quando o estado de conversação é confirmado, o bot executa no chat os caracteres correspondentes ao remetente que nesse caso é o receptor. Finalmente após os tópicos das conversas o bot envia ao usuário uma mensagem de chat representando uma locução em linguagem natural e a conversação atinge o seu estado final.

Como mencionado o conhecimento representa a linguagem encapsulada no tópico. Além disso o gerente de conversação no caso o pandorabots, é capaz de controlar o fluxo de conversação. Especificamente nós incluímos as tags AIML localizadas dentro do template.

\section{Discussão}

Nesta seção a usabilidade do mecanismo proposto é avaliada baseada na conversação do agente. Especificamente os testes foram realizados para avaliar o protótipo construído em um cenário de um Museu Virtual em 3D implementando um agente conversacional. Os objetivos são definidos e a metodologia do teste é descrita, após a descrição dos resultados. Objetivos do teste: $\mathrm{O}$ objetivo principal do teste é avaliar a usabilidade proposta em ambiente imersivo em 3D. Para fazer isso vamos nos concentrar em diferentes critérios de usabilidade como a eficácia, eficiência, erros e satisfação com a abordagem apresentada. Como isso afeta a experiência do usuário com ambientes virtuais e sistemas baseados em conversação. Além disso, este estudo nos permite detectar problemas na estrutura e no conteúdo abordado no Museu Virtual.

\section{Considerações Finais}

Este artigo teve como objetivo apresentar o desenvolvimento de um agente de software, imerso em um mundo virtual 3D, para atuar como guia conversacional em um Museu Virtual da Computação. No desenvolvimento do AGIMC, foi realizada a integração de um chatterbot simulando um agente inteligente conversacional, no intuito de fornecer ao aluno um ambiente de conhecimentos e que auxiliasse nas dúvidas referentes à história e introdução a computação. Para isso foi necessário desenvolver uma base de conhecimento adequada para o tema. 
VII Congresso Brasileiro de Informática na Educação (CBIE 2018)

Anais dos Workshops do VII Congresso Brasileiro de Informática na Educação (WCBIE 2018)

A utilização de ferramentas como o servidor público do pandorabots facilitaram o desenvolvimento do produto e o uso do software com a interconexão do agente inteligente foi necessária, podendo assim verificar se a solução proposta era capaz de atingir os objetivos do trabalho. Diante do exposto, tornou-se possível verificar as simulações de diálogo com o agente e perceber se o diálogo era motivador para estimular os alunos a continuar interagindo, identificando associações com mundo real.

Conforme a avaliação dos resultados foi possível observar que os índices indicam que no momento da avaliação os alunos se sentiram dialogando com o agente, tornando assim o aprendizado mais motivador, continuando o diálogo e querendo mais interação sobre o assunto. A utilização do tema introdução à computação para os alunos de um curso de Licenciatura em Computação favoreceu o diálogo, por terem interesse em conhecer mais sobre a área principalmente por ser um ambiente simulado em um mundo virtual.

A inclusão do agente inteligente facilitou, portanto, o uso do diálogo no museu da computação em que o aluno pode reconhecer o objeto em 3D e poder usar mais da interação, além de possibilitar a utilização do software em sala de aula em aulas de introdução a computação ou cursos que seguem essa metodologia de ensino. A utilização do agente possibilitou uma melhor interação com o aluno, fazendo com que o mesmo se sentisse mais motivado ao diálogo, o que foi observado durante a avaliação. Outra característica do AGIMC foi à disponibilidade, pois ele foi ativo como um bot permitindo que o aluno tire suas dúvidas dialogue e obtenha uma resposta para uma pergunta no horário que lhe convier, ou seja, ele poderá acessar o museu virtual em horários fora da sala de aula e obter o conhecimento usando o mundo virtual como um complemento da disciplina. Como trabalho futuro pretende-se utilizar sensores para investigar a atenção e os estilos de aprendizagem dos estudantes.

\section{Referências}

Carmo, F. M. (2013). Mundo virtual 3D em plataforma aberta como interface para ambientes de aprendizagem. Dissertação de Mestrado na Escola Politécnica da Universidade de São Paulo .

Graesser A. C., P. Chipman, B. C. Haynes, and A. Olney. Auto tutor: An intelligent tutoring system with mixed-initiative dialogue. Education, IEEE Transactions on, 48(4):612-618, 2005.

Galvão A. M.; F. A. Barros, A. M. Neves, and G. L. Ramalho. Persona-aiml: An architecture developing chatterbots with personality. In Proceedings of the Third International Joint Conference on Autonomous Agents and Multiagent SystemsVolume 3, pages 1266-1267. IEEE Computer Society, 2004.

Guomin, Z.; Jianxin, Z.; Na Education Value Analysis of SLoodle-based Distribuidet Virtual Learning System. In: Second International Workshop on Education Technology and Computer Science, IEEE, 2010

Mahapatra, R. P.; Sharma, N.; Trivedi, A.; Aman, C (2012). Adding interactive interface to E-Government systems using AIML based chatterbots. In: Proceedings of Sixth International Conference on Software Engineering (CONSEG 2012), pp.1-6, Sept. 2012. 
VII Congresso Brasileiro de Informática na Educação (CBIE 2018)

Anais dos Workshops do VII Congresso Brasileiro de Informática na Educação (WCBIE 2018)

Mennecke, B.E., Roche, M.D., Bray E. M., Townsend D.A., Lester A. M. (2008), "Second Life and Other Virtual Worlds: A Roadmap for Research", Communications of the Association for Information Systems, 22, 371-388

Morgado, L. Características e desafios tecnológicos dos mundos virtuais no ensino. 2011. 82p. Habilitation Seminar — Universidade de Trás-os-Montes e Alto Douro.

OpenSim (2018) "Opensimulator". Disponível em: opensimulator.org

Open Wonderland: Open source 3D virtual collaboration toolkit, (2018). Disponível em: openwonderland.org /.

Russel, S.; Norvig, P. Inteligência Artificial. 2. ed. Rio de Janeiro: Elsevier Editora, 2004.

Second Life 2018 Disponível em http://www.secondlife.com

Russel, S.; Norvig, P. Inteligência Artificial. 2. ed. Rio de Janeiro: Elsevier Editora, 2004.

Wallace, Richard S. AIML Overview. 2018. Disponível em: http://www.pandorabots.com/pandora/pics/wallaceaimltutorial.html. Acesso em: setembro 2018.

Wooldridge, M. (2009) “An Introduction to Multiagent Systems". Ed. Wiley, England, Second Edition. 